\title{
Stabilization of Semi-Markovian Jump Systems with Uncertain Probability Intensities and Its Extension to Quantized Control
}

\author{
Ngoc Hoai An Nguyen, ${ }^{1}$ Sung Hyun Kim, ${ }^{1}$ and Jun Choi ${ }^{2}$ \\ ${ }^{1}$ School of Electrical Engineering, University of Ulsan, Daehak-ro 93, Nam-Gu, Ulsan 680-749, Republic of Korea \\ ${ }^{2}$ Korea Institute of Industrial Technology, Jongga-ro 55, Jung-gu, Ulsan 44413, Republic of Korea \\ Correspondence should be addressed to Sung Hyun Kim; ie222.kim@gmail.com
}

Received 19 July 2016; Accepted 1 November 2016

Academic Editor: Haitao Zhang

Copyright (c) 2016 Ngoc Hoai An Nguyen et al. This is an open access article distributed under the Creative Commons Attribution License, which permits unrestricted use, distribution, and reproduction in any medium, provided the original work is properly cited.

\begin{abstract}
This paper concentrates on the issue of stability analysis and control synthesis for semi-Markovian jump systems (S-MJSs) with uncertain probability intensities. Here, to construct a more applicable transition model for S-MJSs, the probability intensities are taken to be uncertain, and this property is totally reflected in the stabilization condition via a relaxation process established on the basis of time-varying transition rates. Moreover, an extension of the proposed approach is made to tackle the quantized control problem of S-MJSs, where the infinitesimal operator of a stochastic Lyapunov function is clearly discussed with consideration of input quantization errors.
\end{abstract}

\section{Introduction}

Over the past few decades, considerable attention has been paid to Markovian jump systems (MJSs) since such systems are suitable for representing a class of dynamic systems subject to random abrupt variations. In addition to the growing interest from their representation ability, MJSs have been widely applied in many practical applications, such as manufacturing systems, aircraft control, target tracking, robotics, networked control systems, solar receiver control, and power systems (see [1-9] and references therein). Following this trend, numerous investigations are underway to deal with the issue of stability analysis and control synthesis for MJSs with complete/incomplete knowledge of transition probabilities in the framework of filter and control design problems: [1013] with a complete description of transition rates and [1420 ] without a complete description. Generally, in MJSs, the sojourn-time is given as a random variable characterized by the continuous exponential probability distribution, which tends to make the transition rates time-invariant due to the memoryless property of the probability distribution. The thing to be noticed here is that the use of constant transition rates plays a limited role in representing a wide range of application systems (see [21-23]). Thus, another interesting topic has recently been studied in semi-Markovian jump systems (S-MJSs) to overcome the limitation of this memoryless property.

As reported in [24-26], the mode transition of S-MJSs is driven by a continuous stochastic process governed by the nonexponential sojourn-time distribution, which leads to the appearance of time-varying transition rates. Thus, it has been well recognized that S-MJSs are more general than MJSs in real situations. Further, with this growing recognition, various problems on S-MJSs have been widely studied for successful utilization of a variety of practical applications (see [21, 22, 25, 27-29] and references therein). Of them, the first attempt to overcome the limits of MJSs was made by $[21,22]$ for the stability analysis of systems with phasetype $(\mathrm{PH})$ semi-Markovian jump parameters, which was extended to the state estimation and sliding mode control by [29]. Besides, [25] considered the Weibull distribution for the stability analysis of S-MJSs and introduced a sojourntime partition technique to make the derived stability criterion less conservative. Continuing this, [28] applied the sojourn-time partition technique to the design of $\mathscr{H}_{\infty}$ statefeedback control for S-MJSs with time-varying delays. After 
that, another partition technique of dividing the range of transition rates was proposed by [27] to derive the stability and stabilization conditions of S-MJSs with norm-bounded uncertainties. Most recently, [30] designed a reliable mixed passive and $\mathscr{H}_{\infty}$ filter for semi-Markov jump delayed systems with randomly occurring uncertainties and sensor failures. Also, [26] considered semi-Markovian switching and random measurement while designing a sliding mode control for networked control systems (NCSs). Based on the above observations, it can be found that their key issue mainly lies in finding more applicable transition models for S-MJSs, capable of a broad range of cases. In this light, one needs to explore the impacts of uncertain probability intensities in the study of S-MJSs and then provide a relaxed stability criterion absorbing the property of the resultant time-varying transition rates. However, until now, there have been almost no studies that intensively establish a kind of relaxation process corresponding to the stabilization problem of S-MJSs with uncertain probability intensities.

This paper addresses the issue of stability analysis and control synthesis for S-MJSs with uncertain probability intensities. One of our main contributions is to discover more reliable and scalable transition models for S-MJSs on the basis of their time-varying and boundary properties. To this end, this paper provides a valuable theoretical approach of constructing practical transition models for S-MJSs (1) by taking into account uncertain probability intensities and (2) by reflecting their available bounds in the transition rate description. Further, in a different manner from other works, all constraints on time-varying transition rates are totally incorporated into the stabilization condition via a relaxation process established on the basis of time-varying transition rates. Here, it is worth noticing that our relaxation process is developed in such a way that all possible slack variables can be included therein. In contrast to other works, our relaxation process plays a key role in obtaining a finite and solvable set of linear matrix inequalities (LMIs) from parameterized matrix inequalities (PLMIs) arising from uncertain probability intensities. On the other hand, the quantization module that converts real-valued measurement signals into piecewise constant ones has been commonly used to implement a variety of networked control systems over wired or wireless communications (see $[31,32]$ ). Especially among optical wireless communications, the visible light communication can be applied as a data communication channel to transmit the control input to the S-MJSs under consideration. Thus, as an extension, this paper tackles the quantized control problem of S-MJSs, where the infinitesimal operator of a stochastic Lyapunov function is clearly discussed with consideration on input quantization errors. In addition, this paper proposes a method for reducing the influence of input quantization errors in the control of SMJSs, which is also one of our main contributions. Finally, simulation examples show the effectiveness of the proposed method.

Notation. The notations $X \geq Y$ and $X-Y$ means that $X-Y$ is positive semidefinite and positive definite, respectively. In symmetric block matrices, $(*)$ is used as an ellipsis for terms induced by symmetry. For any square matrix $Q \mathcal{Q}, \mathbf{H e}[Q]=Q+$ $Q^{T}$. For $\mathbb{N}_{s}^{+} \triangleq\{1,2, \ldots, s\}$,

$$
\begin{aligned}
& {\left[Q_{i}\right]_{i \in \mathbb{N}_{s}^{+}} \triangleq\left[Q_{1} Q_{2} \cdots Q_{s}\right],} \\
& {\left[\mathbb{Q}_{i j}\right]_{i, j \in \mathbb{N}_{s}^{+}} \triangleq\left[\begin{array}{ccc}
\mathscr{Q}_{11} & \cdots & \mathscr{Q}_{1 s} \\
\vdots & \ddots & \vdots \\
\mathcal{Q}_{s 1} & \cdots & Q_{s s}
\end{array}\right] \text {, }} \\
& {\left[Q_{i}\right]_{i \in \mathbb{N}_{s}^{+}}^{\mathrm{D}} \triangleq\left[\begin{array}{cccc}
Q_{1} & 0 & \cdots & 0 \\
0 & Q_{2} & \ddots & \vdots \\
\vdots & \ddots & \ddots & 0 \\
0 & \cdots & 0 & \mathbb{Q}_{s}
\end{array}\right]} \\
& {\left[\mathbb{Q}_{i j}\right]_{i, j \in \mathbb{N}_{s}^{+}}^{\mathrm{U}} \triangleq\left[\begin{array}{ccccc}
0 & Q_{12} & Q_{13} & \cdots & Q_{1 s} \\
0 & 0 & Q_{23} & \cdots & Q_{2 s} \\
\vdots & \ddots & \ddots & \ddots & \vdots \\
0 & \cdots & \cdots & 0 & Q_{(s-1) s} \\
0 & \cdots & \cdots & 0 & 0
\end{array}\right] \text {, }}
\end{aligned}
$$

where $Q_{i}$ and $Q_{i j}$ denote real submatrices with appropriate dimensions or scalar values. The notation $\mathbf{E}[\bullet]$ denotes the mathematical expectation and $\operatorname{diag}(\bullet)$ stands for a blockdiagonal matrix. The notation $\lambda_{\max }(\bullet)$ denotes the maximum eigenvalue of the argument, and $\exp (\bullet)$ indicates the exponential distribution.

\section{System Description}

Let us consider the following continuous-time semi-Markovian jump linear systems (S-MJSs):

$$
\dot{x}(t)=A(\zeta(t)) x(t)+B(\zeta(t)) u(t),
$$

where $x(t) \in \mathbb{R}^{n_{x}}$ and $u(t) \in \mathbb{R}^{n_{u}}$ denote the state and the control input, respectively. Here, $\{\zeta(t), t \geq 0\}$ denotes a continuous-time semi-Markov process that takes values in the finite space $\mathbb{N}_{s}^{+}$and further has the mode transition probabilities:

$$
\begin{aligned}
& \operatorname{Pr}(\zeta(t+h)=j \mid \zeta(t)=i) \\
& \quad= \begin{cases}\pi_{i j}(h) h+o(h), & \text { if } j \neq i \\
1+\pi_{i i}(h) h+o(h), & \text { if } j=i,\end{cases}
\end{aligned}
$$

where $\lim _{h \rightarrow 0}(o(h) / h)=0$ and $\pi_{i j}(h)$ denotes the transition rate from mode $i$ to mode $j$ at time $t+h$. Further, $h$ indicates the sojourn-time elapsed when the system stays at mode $i$ from the last jump (i.e., $h$ is set to 0 when the system jumps). 
In particular, the transition rate matrix $\prod(h) \triangleq\left[\pi_{i j}(h)\right]_{i, j \in \mathbb{N}_{s}^{+}}$ belongs to the following set:

$$
\begin{aligned}
& \mathcal{S}_{\Pi}^{(1)} \triangleq\left\{\left[\pi_{i j}\right]_{i, j \in \mathbb{N}_{s}^{+}} \mid 0=\sum_{j \in \mathbb{N}_{s}^{+}} \pi_{i j}, 0\right. \\
& \leq \mu_{i j} \pi_{i j}, \text { where }\left.\mu_{i j}\right|_{j \neq i}=1,\left.\mu_{i j}\right|_{j=i}=-1, \forall i, j \\
& \left.\in \mathbb{N}_{s}^{+}\right\} .
\end{aligned}
$$

Before going ahead, for later convenience, we define the system matrix for the $i$ th mode as $\left(A_{i}, B_{i}\right) \triangleq\left(A\left(\zeta_{k}=i\right), B\left(\zeta_{k}=\right.\right.$ $i)$ ), and set $\prod_{i}(h) \triangleq\left[\begin{array}{lll}\pi_{i 1}(h) & \cdots & \pi_{i s}(h)\end{array}\right]^{T}=\left[\pi_{i j}(h)\right]_{j \in \mathbb{N}_{s}^{+}}^{T}$. Also, to deal with the stability analysis problem in such a stochastic setting, we consider the following definition.

Definition 1. An S-MJS (2) with $u(t)=0$ is stochastically stable if its solution is such that, for any initial condition $x_{0}$ and $\zeta_{0}$,

$$
\lim _{t \rightarrow \infty} \mathbf{E}\left[\int_{0}^{t}\|x(\tau)\|^{2} d \tau \mid x_{0}, \zeta_{0}\right]<\infty
$$

\section{Stochastic Stability Analysis}

First of all, let us consider (2) with $u(t) \equiv 0$ :

$$
\dot{x}(t)=A(\zeta(t)) x(t) .
$$

The following lemma presents the stochastic stability condition for (6) with $\prod(h) \in \mathcal{S}_{\Pi}^{(1)}$.

Lemma 2. Suppose that there exists $P_{i}>0$, for all $i \in \mathbb{N}_{s}^{+}$, such that

$$
\begin{array}{r}
0>Q_{i}(h), \\
\prod(h) \in \delta_{\Pi}^{(1)},
\end{array}
$$

$$
\forall i \in \mathbb{N}_{s}^{+}
$$

where $Q_{i}(h) \triangleq \operatorname{He}\left(P_{i} A_{i}\right)+\sum_{j=1}^{s} \pi_{i j}(h) P_{j}$. Then, S-MJSs (6) with $\prod(h) \in \mathcal{S}_{\Pi}^{(1)}$ are stochastically stable.

Proof. Let us consider a stochastic Lyapunov function candidate of the following form:

$$
V(x(t), \zeta(t))(=V(t))=x^{T}(t) P(\zeta(t)) x(t),
$$

where $P(\zeta(t))$ is taken to be positive definite. Then, the infinitesimal generator $\nabla$ of the stochastic process $\{x(t), \zeta(t), t \geq 0\}$ acting on $V(t)$ is given by

$$
\begin{aligned}
\nabla V(t) & =\lim _{\Delta \rightarrow 0} \frac{\mathbf{E}[V(x(t+\Delta), \zeta(t+\Delta)=j \mid x(t), \zeta(t)=i)]-V(x(t), r(t))}{\Delta} \\
& =\lim _{\Delta \rightarrow 0} \frac{1}{\Delta}\left[\sum_{j=1}^{s} p_{i j}(t, \Delta) x^{T}(t+\Delta) P_{j} x(t+\Delta)-x^{T}(t) P_{i} x(t)\right]=\Psi_{1}(t)+\Psi_{2}(t)+\Psi_{3}(t),
\end{aligned}
$$

where $p_{i j}(t, \Delta)=\operatorname{Pr}(\zeta(t+\Delta)=j \mid \zeta(t)=i)$,

$$
\begin{aligned}
& \Psi_{1}(t) \triangleq \lim _{\Delta \rightarrow 0} \frac{1}{\Delta} \sum_{j=1, j \neq i}^{s} p_{i j}(t, \Delta) x^{T}(t+\Delta) P_{j} x(t+\Delta), \\
& \Psi_{2}(t) \triangleq \lim _{\Delta \rightarrow 0} \frac{1}{\Delta} p_{i i}(t, \Delta) x^{T}(t+\Delta) P_{i} x(t+\Delta), \\
& \Psi_{3}(t) \triangleq \lim _{\Delta \rightarrow 0} \frac{1}{\Delta}\left(-x^{T}(t) P_{i} x(t)\right) .
\end{aligned}
$$

Here, the probabilities $p_{i j}(t, \Delta)$ and $p_{i i}(t, \Delta)$ are described as

$$
\begin{aligned}
p_{i j}(t, \Delta) & =\frac{\operatorname{Pr}(\zeta(t+\Delta)=j, \zeta(t)=i)}{\operatorname{Pr}(\zeta(t)=i)} \\
& =\frac{q_{i j}\left(G_{i}(h+\Delta)-G_{i}(h)\right)}{1-G_{i}(h)}, \quad \forall j \neq i,
\end{aligned}
$$

$$
\begin{aligned}
p_{i i}(t, \Delta) & =\frac{\operatorname{Pr}(\zeta(t+\Delta)=i, \zeta(t)=i)}{\operatorname{Pr}(\zeta(t)=i)} \\
& =\frac{1-G_{i}(h+\Delta)}{1-G_{i}(h)},
\end{aligned}
$$

where $G_{i}(h)$ denotes the cumulative distribution function of the sojourn-time $h$ at mode $i$ and $q_{i j}$ stands for the probability intensity such that $\sum_{j=1, j \neq i}^{s} q_{i j}=1$ and $q_{i i}=-1$. Especially in (11), the probability intensity $q_{i j}$ is taken to have uncertainties. Meanwhile, from (6), it follows that $x(t+\Delta)=\left(A_{i} \Delta+I\right) x(t)$, for $\Delta \rightarrow 0$, which leads to

$$
\begin{aligned}
& x^{T}(t+\Delta) P_{j} x(t+\Delta) \\
& \quad=x^{T}(t)\left(A_{i} \Delta+I\right)^{T} P_{j}\left(A_{i} \Delta+I\right) x(t) \\
& \quad=x^{T}(t)\left(\Delta^{2} \cdot A_{i}^{T} P_{j} A_{i}+\Delta \cdot \operatorname{He}\left(P_{j} A_{i}\right)+P_{j}\right) x(t) .
\end{aligned}
$$


That is, from (11) and (12), it is clear that

$$
\begin{gathered}
\lim _{\Delta \rightarrow 0} \frac{1}{\Delta} p_{i j}(t, \Delta) x^{T}(t+\Delta) P_{j} x(t+\Delta) \\
=\lim _{\Delta \rightarrow 0} p_{i j}(t, \Delta) x^{T}(t) \operatorname{He}\left(P_{j} A_{i}\right) x(t) \\
\quad+\lim _{\Delta \rightarrow 0} \frac{1}{\Delta} p_{i j}(t, \Delta) x^{T}(t) P_{j} x(t) .
\end{gathered}
$$

Thus, using (14), $\Psi_{1}(t)$ and $\Psi_{2}(t)$ become

$$
\begin{aligned}
\Psi_{1}(t)= & \sum_{j=1, j \neq i}^{s} \lim _{\Delta \rightarrow 0} p_{i j}(t, \Delta) x^{T}(t) \mathbf{H e}\left(P_{i} A_{i}\right) x(t) \\
& +\lim _{\Delta \rightarrow 0} \frac{1}{\Delta} \sum_{j=1, j \neq i}^{s} p_{i j}(t, \Delta) x^{T}(t) P_{j} x(t) \\
= & \lim _{\Delta \rightarrow 0} \frac{1}{\Delta} \sum_{j=1, j \neq i}^{s} p_{i j}(t, \Delta) x^{T}(t) P_{j} x(t), \\
\Psi_{2}(t)= & \lim _{\Delta \rightarrow 0} p_{i i}(t, \Delta) x^{T}(t) \mathbf{H e}\left(P_{i} A_{i}\right) x(t) \\
& +\lim _{\Delta \rightarrow 0} \frac{1}{\Delta} p_{i i}(t, \Delta) x^{T}(t) P_{i} x(t) \\
= & x^{T}(t) \operatorname{He}\left(P_{i} A_{i}\right) x(t) \\
& +\lim _{\Delta \rightarrow 0} \frac{1}{\Delta} p_{i i}(t, \Delta) x^{T}(t) P_{i} x(t) .
\end{aligned}
$$

That is, $\nabla V(t)$ becomes

$$
\begin{aligned}
& \nabla V(t)=\lim _{\Delta \rightarrow 0} \frac{1}{\Delta} \sum_{j=1, j \neq 1}^{s} p_{i j}(t, \Delta) x^{T}(t) P_{j} x(t)+x^{T}(t) \\
& \cdot \operatorname{He}\left(P_{i} A_{i}\right) x(t) \\
& +\lim _{\Delta \rightarrow 0} \frac{1}{\Delta}\left(p_{i i}(t, \Delta)-1\right) x^{T}(t) P_{i} x(t)=x^{T}(t) \\
& \cdot\left\{\operatorname{He}\left(P_{i} A_{i}\right)\right. \\
& \left.+\lim _{\Delta \rightarrow 0} \frac{1}{\Delta}\left(\sum_{j=1, j \neq i}^{s} p_{i j}(t, \Delta) P_{j}+\left(p_{i i}(t, \Delta)-1\right) P_{i}\right)\right\} \\
& \cdot x(t)=x^{T}(t)\left\{\operatorname{He}\left(P_{i} A_{i}\right)\right. \\
& \left.+\lim _{\Delta \rightarrow 0} \frac{1}{\Delta}\left(\sum_{j=1, j \neq i}^{s} q_{i j} P_{j}-P_{i}\right) \frac{G_{i}(h+\Delta)-G_{i}(h)}{1-G_{i}(h)}\right\} \\
& \cdot x(t)=x^{T}(t)\left\{\operatorname{He}_{i}\left(P_{i} A_{i}\right)\right.
\end{aligned}
$$

$$
\begin{aligned}
& \left.+\left(\sum_{j=1, j \neq i}^{s} q_{i j} P_{j}-P_{i}\right) \frac{g_{i}(h)}{1-G_{i}(h)}\right\} x(t)=x^{T}(t) \\
& \cdot\left\{\operatorname{He}\left(P_{i} A_{i}\right)+\left(\sum_{j=1, j \neq i}^{s} q_{i j} P_{j}-P_{i}\right) \pi_{i}(h)\right\} x(t),
\end{aligned}
$$

where $\pi_{i}(h)=g_{i}(h) / 1-G_{i}(h)$ denotes the transition rate of the system jumping from mode $i$. As a result, by defining $\pi_{i j}(h)=$ $q_{i j} \pi_{i}(h)$, for $j \neq i$, and $\pi_{i i}(h)=-\sum_{j=1, j \neq i}^{s} \pi_{i j}(h)=-\pi_{i}(h)$, we have

$$
\begin{aligned}
& \nabla V(t)=x^{T}(t) \\
& \cdot\left\{\operatorname{He}\left(P_{i} A_{i}\right)+\sum_{j=1, j \neq i}^{s} \pi_{i j}(h) P_{j}+\pi_{i i}(h) P_{i}\right\} x(t) \\
& =x^{T}(t) Q_{i}(h) x(t) .
\end{aligned}
$$

In what follows, by the generalized Dynkin's formula [33], it is clear that

$$
\begin{aligned}
\mathbf{E}[V(t)]-V(0)= & \mathbf{E}\left[\int_{0}^{t} \nabla V(\tau) d \tau \mid x_{0}, \zeta_{0}\right] \\
\leq & \max _{i \in \mathbb{N}_{s}^{+}, 0 \leq h \leq t}\left(\lambda_{\max }\left(Q_{i}(h)\right)\right) \\
& \cdot \mathbf{E}\left[\int_{0}^{t}\|x(\tau)\|_{2}^{2} d \tau \mid x_{0}, \zeta_{0}\right],
\end{aligned}
$$

which results in

$$
\begin{aligned}
& -\max _{i \in \mathbb{N}_{s}^{+}, 0 \leq h \leq t}\left(\lambda_{\max }\left(Q_{i}(h)\right)\right) \\
& \cdot \mathbf{E}\left[\int_{0}^{t}\|x(\tau)\|_{2}^{2} d \tau \mid x_{0}, \zeta_{0}\right] \leq V(0)-\mathbf{E}[V(t)] \\
& \quad \leq V(0) .
\end{aligned}
$$

Thus, from (7), it follows that

$$
\begin{aligned}
& \lim _{t \rightarrow \infty} \mathbf{E}\left[\int_{0}^{t}\|x(\tau)\|_{2}^{2} d \tau \mid x_{0}, \zeta_{0}\right] \\
& \quad \leq-\frac{V(0)}{\max _{i \in \mathbb{N}_{s}^{+}, 0 \leq h}\left(\lambda_{\max }\left(Q_{i}(h)\right)\right)}<\infty .
\end{aligned}
$$

Finally, by Definition 1, the proof can be completed.

In this paper, as a model of probability distribution for the sojourn-time $h \geq 0$, we utilize the Weibull distribution with shape parameter $\beta>0$ and scale parameter $\alpha>0$, since such a distribution has been witnessed as an appropriate choice for representing the stochastic behavior of practical systems. In other words, to represent the probability distribution of 
$h$, its cumulative function $G_{i}(h)$ and probability distribution function $g_{i}(h)$ are given as follows: for all $i$ and $j(j \neq i) \in \mathbb{N}_{s}^{+}$,

$$
\begin{aligned}
& G_{i}(h)=1-\exp \left(-\left(\frac{h}{\alpha_{i}}\right)^{\beta_{i}}\right), \\
& g_{i}(h)=\frac{\beta_{i}}{\alpha_{i}^{\beta_{i}}} h^{\beta_{i}-1} \exp \left(-\left(\frac{h}{\alpha_{i}}\right)^{\beta_{i}}\right),
\end{aligned}
$$

which leads to

$$
\pi_{i j}(h)=q_{i j} \pi_{i}(h)=q_{i j} \frac{g_{i}(h)}{1-G_{i}(h)}=q_{i j} \frac{\beta_{i}}{\alpha_{i}^{\beta_{i}}} h^{\beta_{i}-1} .
$$

As a special case, let $\beta_{i}=1$. Then, we can represent MJSs from (22); that is, the transition rate $\pi_{i j}(h)$ can be reduced to an $h$-independent value as follows: $\pi_{i j}(h)=q_{i j} \pi_{i}(h)=q_{i j} / \alpha_{i}$. Accordingly, it can be claimed that (22) expresses a more generalized transition model, compared to the case of MJSs.

Remark 3. As shown in (22), the transition rate $\pi_{i j}(h)$ is timevarying and depends on the probability intensity $q_{i j}$. Thus, to derive a finite number of solvable conditions from (7), there is a need to consider the lower and upper bounds of both $\pi_{i}(h)$ and $q_{i j}$, respectively, as follows: $\pi_{i, 1} \leq \pi_{i}(h) \leq \pi_{i, 2}$ and $q_{i j, 1} \leq$ $q_{i j} \leq q_{i j, 2}$. Then, from $\pi_{i j}(h)=q_{i j} \pi_{i}(h)$, the bounds of $\pi_{i j}(h)$ are decided as follows: $\pi_{i j, 1} \leq \pi_{i j}(h) \leq \pi_{i j, 2}$, where

$$
\begin{aligned}
& \pi_{i j, 1}= \begin{cases}q_{i j, 1} \cdot \pi_{i, 1}, & \text { if } j \neq i \\
-\pi_{i, 2}, & \text { otherwise, }\end{cases} \\
& \pi_{i j, 2}= \begin{cases}q_{i j, 2} \cdot \pi_{i, 2}, & \text { if } j \neq i \\
-\pi_{i, 1}, & \text { otherwise. }\end{cases}
\end{aligned}
$$

In accordance with Remark 3, an auxiliary constraint can be established as follows: $\prod(h) \in \mathcal{S}_{\Pi}^{(2)}$, where

$$
\mathcal{S}_{\Pi}^{(2)} \triangleq\left\{\left[\pi_{i j}\right]_{i, j \in \mathbb{N}_{s}^{+}} \mid \pi_{i j, 1} \leq \pi_{i j} \leq \pi_{i j, 2}, \forall i, j \in \mathbb{N}_{s}^{+}\right\} .
$$

The following lemma presents the stochastic stability condition for S-MJSs (6) with $\prod(h) \in \delta_{\Pi}^{(1)} \cap \delta_{\Pi}^{(2)}$.

Lemma 4. Suppose that there exists $P_{i}>0$, for all $i \in \mathbb{N}_{s}^{+}$, such that

$$
\begin{gathered}
0>Q_{i}(h), \\
\prod(h) \in \mathcal{S}_{\Pi}^{(1)} \cap \delta_{\Pi}^{(2)},
\end{gathered}
$$

$\forall i \in \mathbb{N}_{s}^{+}$

where $Q_{i}(h) \triangleq \operatorname{He}\left(P_{i} A_{i}\right)+\sum_{j=1}^{s} \pi_{i j}(h) P_{j}$ and $\pi_{i j}(h) \in$ $\left[\pi_{i j, 1}, \pi_{i j, 2}\right]$. Then, $S$-MJSs (6) with $\mathcal{S}_{\Pi}^{(1)} \cap \mathcal{S}_{\Pi}^{(2)}$ are stochastically stable.

However, it is worth noticing that solving (25) of Lemma 4 is still equivalent to solving an infinite number of LMIs, which is an extremely difficult problem. Thus, it is necessary to find a finite number of solvable LMI-based conditions from (25). To this end, the following theorem provides a relaxed stochastic stability condition for (6) with $\prod(h) \in \mathcal{S}_{\Pi}^{(1)} \cap \mathcal{S}_{\Pi}^{(2)}$.

Theorem 5. Suppose that there exists matrices $\left\{G_{i}, S_{i j}, X_{i j}\right.$, $\left.Y_{i j}\right\}_{i, j \in \mathbb{N}_{s}^{+}} \in \mathbb{R}^{n_{x} \times n_{x}}$ and symmetric matrices $\left\{P_{i}>0\right\}_{i \in \mathbb{N}_{s}^{+}} \in$ $\mathbb{R}^{n_{x} \times n_{x}}$ such that

$$
\begin{aligned}
& 0>\left[\begin{array}{c|c}
(1,1) & (1,2) \\
\hline(*) & (2,2)
\end{array}\right], \quad \forall i \in \mathbb{N}_{s}^{+}, \\
& 0 \leq \operatorname{He}\left(X_{i j}\right), \quad \forall i, j \in \mathbb{N}_{s}^{+}, \\
& 0 \leq \operatorname{He}\left(Y_{i j}\right), \quad \forall i, j \in \mathbb{N}_{s}^{+},
\end{aligned}
$$

where $\left.\mu_{i j}\right|_{j \neq i}=1,\left.\mu_{i j}\right|_{j=i}=-1$,

$$
\begin{aligned}
(1,1)= & \operatorname{He}\left(P_{i} A_{i}\right)-\sum_{j \in \mathbb{N}_{s}^{+}} \mathbf{H e}\left(\pi_{i j, 1} \pi_{i j, 2} X_{i j}\right), \\
(1,2)= & {\left[\frac{1}{2} P_{j}+G_{i}+\left(\pi_{i j, 1}+\pi_{i j, 2}\right) X_{i j}+\mu_{i j} Y_{i j}\right]_{j \in \mathbb{N}_{s}^{+}} } \\
(2,2)= & {\left[\mathbf{H e}\left(S_{i a}-X_{i a}\right)\right]_{a \in \mathbb{N}_{s}^{+}}^{\mathbf{D}} } \\
& +\mathbf{H e}\left(\left[S_{i a}+S_{i b}\right]_{a, b \in \mathbb{N}_{s}^{+}}^{\mathbf{U}} .\right.
\end{aligned}
$$

Then, S-MJSs (6) with $\prod(h) \in \mathcal{S}_{\Pi}^{(1)} \cap \mathcal{S}_{\Pi}^{(2)}$ are stochastically stable.

Proof. From the first constraint $\prod(h) \in \mathcal{S}_{\Pi}^{(1)}$, we can obtain, under (28),

$$
\begin{aligned}
& 0 \equiv \mathbf{H e}\left(\left(\sum_{j \in \mathbb{N}_{s}^{+}} \pi_{i j}(h)\right)\left(G_{i}+\sum_{j \in \mathbb{N}_{s}^{+}} \pi_{i j}(h) S_{i j}\right)\right), \\
& 0 \leq \sum_{j \in \mathbb{N}_{s}^{+}} \mu_{i j} \pi_{i j} \operatorname{He}\left(Y_{i j}\right), \quad \forall i \in \mathbb{N}_{s}^{+},
\end{aligned}
$$

Further, under (27), the second constraint $\prod(h) \in \mathcal{S}_{\Pi}^{(2)}$ provides

$$
0 \leq-\sum_{j \in \mathbb{N}_{s}^{+}}\left(\pi_{i j}(h)-\pi_{i j, 1}\right)\left(\pi_{i j}(h)-\pi_{i j, 2}\right) \mathbf{H e}\left(X_{i j}\right)
$$

$\forall i \in \mathbb{N}_{s}^{+}$ 
Here, note that (30)-(32) can be converted, respectively, into

$$
\begin{aligned}
0 & \left.\equiv\left[\frac{I}{\prod_{i}(h) \otimes I}\right]^{T}\left[\begin{array}{c|c}
0 & {\left[G_{i}\right]_{i \in \mathbb{N}_{s}^{+}}} \\
\hline(*) & (2,2)^{*}
\end{array}\right] \frac{I}{\prod_{i}(h) \otimes I}\right], \\
0 & \leq\left[\frac{I}{\prod_{i}(h) \otimes I}\right]^{T}\left[\begin{array}{c|c}
0 & {\left[\mu_{i j} Y_{i j}\right]_{j \in \mathbb{N}_{s}^{+}}} \\
\hline(*) & 0
\end{array}\right]\left[\frac{I}{\prod_{i}(h) \otimes I}\right], \\
0 & \leq\left[\frac{I}{\prod_{i}(h) \otimes I}\right]^{T} \\
& \cdot\left[\begin{array}{c|c}
(1,1)^{*} & {\left[\left(\pi_{i j, 1}+\pi_{i j, 2}\right) X_{i j}\right]_{j \in \mathbb{N}_{s}^{+}}} \\
\hline(*) & {\left[\operatorname{He}\left(-X_{i a}\right)\right]_{a \in \mathbb{N}_{s}^{+}}^{\mathrm{D}}}
\end{array}\right]\left[\frac{I}{\prod_{i}(h) \otimes I}\right],
\end{aligned}
$$

where $(1,1)^{*}=-\sum_{j \in \mathbb{N}_{s}^{+}} \operatorname{He}\left(\pi_{i j, 1} \pi_{i j, 2} X_{i j}\right)$ and $(2,2)^{*}=$ $\left[\mathrm{He}\left(S_{i a}\right)\right]_{a \in \mathbb{N}_{s}^{+}}^{\mathrm{D}}+\mathbf{H e}\left(\left[S_{i a}+S_{i b}\right]_{a, b \in \mathbb{N}_{s}^{+}}^{\mathrm{U}}\right)$. Likewise, according to the form of (33)-(35), we can rewrite $\mathbb{Q}_{i}(h)<0$ in Lemma 4 as follows:

$$
\begin{aligned}
& \mathbb{Q}_{i}(h)=\left[\frac{I}{\prod_{i}(h) \otimes I}\right]^{T} \\
& \quad \cdot\left[\begin{array}{c|c}
\mathbf{H e}\left(P_{i} A_{i}\right) & {\left[(1 / 2) P_{j}\right]_{j \in \mathbb{N}_{s}^{+}}}
\end{array}\right]\left[\frac{I}{\prod_{i}(h) \otimes I}\right]<0 .
\end{aligned}
$$

As a result, by combining (36) with (33)-(35) through the Sprocedure [34], the stochastic stability condition (25) is given by

$$
0>\left[\frac{I}{\prod_{i}(h) \otimes I}\right]^{T}\left[\begin{array}{c|c}
(1,1) & (1,2) \\
\hline(*) & (2,2)
\end{array}\right]\left[\frac{I}{\prod_{i}(h) \otimes I}\right],
$$

where $(1,1),(1,2)$, and $(2,2)$ are given in the body of Theorem 5. Therefore, as (26) implies (37), the proof can be completed.

\section{Control Design}

Let us consider the following mode-dependent state-feedback control law:

$$
u(t)=F_{i} x(t)
$$

where $F_{i} \triangleq F(\zeta(t)=i)$. Thereby, the resultant closed-loop system under (2) and (38) is given by

$$
\begin{aligned}
\dot{x}(t) & =A_{i} x(t)+B_{i} u(t)=\left(A_{i}+B_{i} F_{i}\right) x(t) \\
& =\bar{A}_{i} x(t) .
\end{aligned}
$$

The following theorem provides a relaxed stochastic stabilization condition for S-MJSs (39) with $\prod(h) \in \mathcal{S}_{\Pi}^{(1)} \cap \mathcal{S}_{\Pi}^{(2)}$.
Theorem 6. Suppose that there exist matrices $\left\{\bar{F}_{i}\right\}_{i \in \mathbb{N}_{s}^{+}} \in$ $\mathbb{R}^{n_{x} \times n_{x}}$ and $\left\{G_{i}, S_{i j}, X_{i j}, Y_{i j}, Q_{i j}\right\}_{i, j \in \mathbb{N}_{s}^{+}} \in \mathbb{R}^{n_{x} \times n_{x}}$ and symmetric matrices $\left\{\bar{P}_{i}>0\right\}_{i \in \mathbb{N}_{s}^{+}} \in \mathbb{R}^{n_{x} \times n_{x}}$ such that

$$
\begin{aligned}
& 0>\left[\begin{array}{c|c}
(1,1) & (1,2) \\
\hline(*) & (2,2)
\end{array}\right], \quad \forall i \in \mathbb{N}_{s}^{+}, \\
& 0 \leq \operatorname{He}\left(X_{i j}\right), \quad \forall i, j \in \mathbb{N}_{s}^{+}, \\
& 0 \leq \operatorname{He}\left(Y_{i j}\right), \quad \forall i, j \in \mathbb{N}_{s}^{+}, \\
& 0 \leq\left[\begin{array}{cc}
Q_{i j} & \bar{P}_{i} \\
(*) & \bar{P}_{j}
\end{array}\right], \quad \forall i, j \neq i \in \mathbb{N}_{s}^{+},
\end{aligned}
$$

where $\left.\epsilon_{i j}\right|_{j \neq i}=1,\left.\epsilon_{i j}\right|_{j=i}=0,\left.\mu_{i j}\right|_{j \neq i}=1,\left.\mu_{i j}\right|_{j=i}=-1$,

$$
\begin{aligned}
& (1,1)=\operatorname{He}\left(A_{i} \bar{P}_{i}+B_{i} \bar{F}_{i}\right)-\sum_{j \in \mathbb{N}_{s}^{+}} \mathbf{H e}\left(\pi_{i j, 1} \pi_{i j, 2} X_{i j}\right), \\
& (1,2)=\left[\left(\frac{1}{2} \epsilon_{i j} Q_{i j}+\frac{1}{2}\left(1-\epsilon_{i j}\right) \bar{P}_{i}\right)+G_{i}\right. \\
& \left.\quad+\left(\pi_{i j, 1}+\pi_{i j, 2}\right) X_{i j}+\mu_{i j} Y_{i j}\right]_{j \in \mathbb{N}_{s}^{+}} \\
& (2,2)=\left[\operatorname{He}\left(S_{i a}-X_{i a}\right)\right]_{a \in \mathbb{N}_{s}^{+}}^{\mathrm{D}}+\mathbf{H e}\left(\left[S_{i a}+S_{i b}\right]_{a, b \in \mathbb{N}_{s}^{+}}^{\mathrm{U}}\right) .
\end{aligned}
$$

Then, closed-loop system (39) with $\prod(h) \in \mathcal{S}_{\Pi}^{(1)} \cap \mathcal{S}_{\Pi}^{(2)}$ is stochastically stable, where $F_{i}=\bar{F}_{i} \bar{P}_{i}^{-1}$.

Proof. In light of (25), the stabilization condition of (39) is given by

$$
0>\operatorname{He}\left(P_{i} \bar{A}_{i}\right)+\sum_{j=1}^{s} \pi_{i j}(h) P_{j} .
$$

Also, pre- and postmultiplying (45) by $\bar{P}_{i} \triangleq P_{i}^{-1}$ yields

$$
0>\bar{Q}_{i}(h) \triangleq \mathbf{H e}\left(A_{i} \bar{P}_{i}+B_{i} \bar{F}_{i}\right)+\sum_{j=1}^{s} \pi_{i j}(h) \bar{P}_{i} P_{j} \bar{P}_{i},
$$

where $\bar{F}_{i} \triangleq F_{i} \bar{P}_{i}$. Here, by employing $Q_{i j}$ such that (43) holds (i.e., $Q_{i j} \geq \bar{P}_{i} P_{j} \bar{P}_{i}$ ), we can convert (46) into

$$
\begin{aligned}
0> & \bar{Q}_{i}(h) \\
= & \operatorname{He}\left(A_{i} \bar{P}_{i}+B_{i} \bar{F}_{i}\right) \\
& +\sum_{j=1}^{s} \pi_{i j}(h)\left(\epsilon_{i j} Q_{i j}+\left(1-\epsilon_{i j}\right) \bar{P}_{i}\right) .
\end{aligned}
$$

Thereupon, $\bar{Q}_{i}(h)$ is factorized as follows:

$$
\begin{aligned}
& \bar{Q}_{i}(h)=\left[\frac{I}{\prod_{i}(h) \otimes I}\right]^{T} \\
& \quad \cdot\left[\begin{array}{c|c}
\mathbf{H e}\left(A_{i} \bar{P}_{i}+B_{i} \bar{F}_{i}\right) & (1,2)^{*} \\
\hline(*) & 0
\end{array}\right]\left[\begin{array}{c}
I \\
\hline \prod_{i}(h) \otimes I
\end{array},\right.
\end{aligned}
$$

where $(1,2)^{*}=\left[(1 / 2) \epsilon_{i j} Q_{i j}+(1 / 2)\left(1-\epsilon_{i j}\right) \bar{P}_{i}\right]_{j \in \mathbb{N}_{s}^{+}}$. 
Next, as in the proof of Theorem 5, the constraint $\prod(h) \epsilon$ $\mathcal{S}_{\Pi}^{(1)} \cap \mathcal{\delta}_{\Pi}^{(2)}$ can be represented as

$$
\begin{aligned}
& 0 \equiv\left[\begin{array}{c|c}
I \\
\hline \prod_{i}(h) \otimes I
\end{array}\right]^{T}\left[\begin{array}{c|c}
0 & {\left[G_{i}\right]_{i \in \mathbb{N}_{s}^{+}}} \\
\hline(*) & (2,2)^{*}
\end{array}\right]\left[\frac{I}{\prod_{i}(h) \otimes I}\right], \\
& 0 \leq\left[\frac{I}{\prod_{i}(h) \otimes I}\right]^{T}\left[\begin{array}{c|c}
0 & {\left[\mu_{i j} Y_{i j}\right]_{j \in \mathbb{N}_{s}^{+}}} \\
\hline(*) & 0
\end{array}\right]\left[\frac{I}{\prod_{i}(h) \otimes I}\right], \\
& 0 \leq\left[\frac{I}{\prod_{i}(h) \otimes I}\right]^{T} \\
& \cdot\left[\begin{array}{c|c}
(1,1)^{*} & {\left[\left(\pi_{i j, 1}+\pi_{i j, 2}\right) X_{i j}\right]_{j \in \mathbb{N}_{s}^{+}}} \\
\hline(*) & {\left[\operatorname{He}\left(-X_{i a}\right)\right]_{a \in \mathbb{N}_{s}^{+}}^{\mathrm{D}}}
\end{array}\right]\left[\frac{I}{\prod_{i}(h) \otimes I}\right],
\end{aligned}
$$

where $(1,1)^{*}=-\sum_{j \in \mathbb{N}_{s}^{+}} \mathbf{H e}\left(\pi_{i j, 1} \pi_{i j, 2} X_{i j}\right)$ and $(2,2)^{*}=$ $\left[\operatorname{He}\left(S_{i a}\right)\right]_{a \in \mathbb{N}_{s}^{+}}^{\mathrm{D}}+\mathbf{H e}\left(\left[S_{i a}+S_{i b}\right]_{a, b \in \mathbb{N}_{s}^{+}}^{\mathrm{U}}\right)$. As a result, by the Sprocedure, combining (48) with (49) results in

$$
0>\left[\begin{array}{c|c}
I \\
\hline \prod_{i}(h) \otimes I
\end{array}\right]^{T}\left[\begin{array}{c|c}
(1,1) & (1,2) \\
\hline(*) & (2,2)
\end{array}\right]\left[\frac{I}{\prod_{i}(h) \otimes I}\right],
$$

where $(1,1),(1,2)$, and $(2,2)$ are given in the Theorem 6 . Therefore, as (40) implies (50), the proof can be completed.

Hereafter, as a practical extension of the proposed approach, we consider the following input-quantized S-MJSs:

$$
\dot{x}(t)=A_{i} x(t)+B_{i} \mathbf{q}(u(t)),
$$

where $\mathbf{q}(\bullet)$ stands for a uniform quantization operator with the quantization level $\delta>0$; that is, $\mathbf{q}(u(t))=\delta$. $\operatorname{round}(u(t) / \delta)$. Here, note that $\mathbf{q}(u(t))=u(t)+\varphi(t)$, where the $k$ th element of the quantization error $\varphi(t)$ satisfies

$$
\left|\varphi_{k}(t)\right| \leq \frac{\delta}{2}, \quad \forall k \in \mathbb{N}_{n_{u}}^{+}
$$

Thus, (51) can be rewritten as

$$
\dot{x}(t)=A_{i} x(t)+B_{i}(u(t)+\varphi(t)),
$$

where $\varphi(t)=\mathbf{q}(u(t))-u(t)$ is known. Continuously, as a mode-dependent state-feedback law, we adopt

$$
u(t)=F_{i} x(t)+v_{i}(t) .
$$

Then, the resultant closed-loop system is described as

$$
\dot{x}(t)=\bar{A}_{i} x(t)+B_{i}\left(v_{i}(t)+\varphi(t)\right) .
$$

The following theorem provides a relaxed stochastic stabilization condition for S-MJSs (55) with input quantization error.

Theorem 7. Let $v_{i, k}$ (i.e., the kth element of $v_{i}$ ) be given as follows:

$$
\begin{aligned}
v_{i, k}(t)= & -\delta \cdot \operatorname{sgn}\left(s_{i, k}(t)\right) \\
& \cdot \max \left(0, \operatorname{sgn}\left(s_{i}^{T}(t) \varphi(t)\right)\right),
\end{aligned}
$$

where $s_{i}(t)=B_{i}^{T} P_{i} x(t) \in \mathbb{R}^{n_{u}}$ and $s_{i, k}(t)$ denotes the $k t h$ element of $s_{i}(t)$. Suppose that there exist matrices $\left\{\bar{F}_{i}\right\}_{i \in \mathbb{N}_{s}^{+}} \in$ $\mathbb{R}^{n_{x} \times n_{x}}$ and $\left\{G_{i}, S_{i j}, X_{i j}, Y_{i j}, Q_{i j}\right\}_{i, j \in \mathbb{N}_{s}^{+}} \in \mathbb{R}^{n_{x} \times n_{x}}$ and symmetric matrices $\left\{\bar{P}_{i}>0\right\}_{i \in \mathbb{N}_{s}^{+}} \in \mathbb{R}^{n_{x} \times n_{x}}$ such that (40)-(43) hold. Then, the closed-loop system (55) with $\prod(h) \in \mathcal{S}_{\Pi}^{(1)} \cap \mathcal{S}_{\Pi}^{(2)}$ is stochastically stable, where $F_{i}=\bar{F}_{i} \bar{P}_{i}^{-1}$.

Proof. From (55), it follows that $x(t+\Delta)=\left(\bar{A}_{i} \Delta+I\right) x(t)+$ $B_{i} \Delta\left(\nu_{i}(t)+\varphi(t)\right)$, for $\Delta \rightarrow 0$, which yields

$$
\begin{aligned}
x^{T}(t+\Delta) P_{j} x(t+\Delta) & \\
= & \cdot x^{T}(t) \operatorname{He}\left(P_{j} \bar{A}_{i}\right) x(t)+x^{T}(t) P_{j} x(t)+\Delta \\
& \cdot 2 x^{T}(t) P_{j} B_{i}\left(v_{i}(t)+\varphi(t)\right)+\Delta^{2} \cdot(\bullet) .
\end{aligned}
$$

Thus, it is given that

$$
\begin{aligned}
\lim _{\Delta \rightarrow 0} & \frac{1}{\Delta} p_{i j}(t, \Delta) x^{T}(t+\Delta) P_{j} x(t+\Delta)=\lim _{\Delta \rightarrow 0} p_{i j}(t, \Delta) \\
\cdot & \left(x^{T}(t) \operatorname{He}\left(P_{j} \bar{A}_{i}\right) x(t)\right. \\
& \left.+2 x^{T}(t) P_{j} B_{i}\left(v_{i}(t)+\varphi(t)\right)\right)+\lim _{\Delta \rightarrow 0} \frac{1}{\Delta} p_{i j}(t, \Delta) \\
& \cdot x^{T}(t) P_{j} x(t) .
\end{aligned}
$$

Then, based on (11) and (12), we have

$$
\begin{aligned}
\Psi_{1}(t) & =\sum_{j=1, j \neq i}^{s} \lim _{\Delta \rightarrow 0} \frac{1}{\Delta} p_{i j}(t, \Delta) x^{T}(t) P_{j} x(t), \\
\Psi_{2}(t) & +\Psi_{3}(t) \\
= & x^{T}(t) \operatorname{He}\left(P_{i} \bar{A}_{i}\right) x(t) \\
& +2 x^{T}(t) P_{i} B_{i}\left(\nu_{i}(t)+\varphi(t)\right) \\
& +\lim _{\Delta \rightarrow 0} \frac{1}{\Delta}\left(p_{i i}(t, \Delta)-1\right) x^{T}(t) P_{i} x(t),
\end{aligned}
$$

which leads to

$$
\begin{aligned}
\nabla V(t)= & x^{T}(t)\left(\operatorname{He}\left(P_{i} \bar{A}_{i}\right)+\sum_{j=1}^{s} \pi_{i j}(h) P_{j}\right) x(t) \\
& +2 s_{i}^{T}(t)\left(\nu_{i}(t)+\varphi(t)\right) \\
s_{i}(t)= & B_{i}^{T} P_{i} x(t) \in \mathbb{R}^{n_{u}} .
\end{aligned}
$$

Hence, for $s_{i}^{T}(t) \varphi(t) \leq 0$, letting $\nu_{i}(t) \equiv 0$ implies

$$
\nabla V(t) \leq x^{T}(t)\left(\mathbf{H e}\left(P_{i} \bar{A}_{i}\right)+\sum_{j=1}^{s} \pi_{i j}(h) P_{j}\right) x(t) .
$$


Further, for $s_{i}^{T}(t) \varphi(t)>0$, letting $\nu_{i, k}(t)=-\delta \cdot \operatorname{sgn}\left(s_{i, k}(t)\right)$ yields

$$
\begin{aligned}
2 s_{i}^{T}(t)\left(\nu_{i}(t)+\varphi(t)\right)= & \sum_{k=1}^{n_{u}} 2 s_{i, k}(t)\left(v_{i, k}(t)+\varphi_{k}(t)\right) \\
\leq & \sum_{k=1}^{n_{u}}-2 \delta\left|s_{i, k}(t)\right|+2\left|s_{i, k}(t)\right| \\
& \cdot\left|\varphi_{k}(t)\right| \leq-\sum_{k=1}^{n_{u}} \delta\left|s_{i, k}(t)\right|<0 .
\end{aligned}
$$

That is, (56) allows (61) to be reduced to (62) for all $s_{i}^{T}(t) \varphi(t)$. In what follows, we need to derive the stabilization condition from (62), which is omitted herein because it is in line with the proof of Theorem 6 .

\section{Numerical Examples}

Example 1. Consider the following system with three different modes: for $x_{0}=(3.0,-5.0)$ and $\zeta_{0}=3$,

$$
\begin{aligned}
& A_{1}=\left[\begin{array}{cc}
-0.5 & -0.75 \\
1.00 & 1.00
\end{array}\right], \\
& A_{2}=\left[\begin{array}{cc}
-2.40 & -0.33 \\
1.00 & -1.40
\end{array}\right], \\
& A_{3}=\left[\begin{array}{cc}
-0.20 & 0.10 \\
1.00 & -1.00
\end{array}\right], \\
& B_{1}=\left[\begin{array}{c}
5.0 \\
0.0
\end{array}\right], \\
& B_{2}=\left[\begin{array}{c}
-2.0 \\
-1.0
\end{array}\right], \\
& B_{3}=\left[\begin{array}{c}
1.0 \\
-2.0
\end{array}\right] .
\end{aligned}
$$

Here, the Weibull distribution for the sojourn-time is set by $\left(\alpha_{1}, \beta_{1}\right)=(0.5,2.0),\left(\alpha_{2}, \beta_{2}\right)=(1.0,2.0)$, and $\left(\alpha_{3}, \beta_{3}\right)=$ $(1.5,2.0)$ (see Figure 1). Thereby, a reasonable interval $h \in$ $\left[h_{0}, h_{1}\right]$ can be obtained such that $\int_{h_{0}}^{h_{1}} g_{i}(h) d h \geq 0.99$, from which the lower and upper bounds $\left(\pi_{i, 1}, \pi_{i, 2}\right)$ of $\pi_{i}(h)$ can be also found. As a result, by considering the uncertain probability intensities such that $0.3 \leq q_{i j} \leq 0.7$, for all $i$ and $j$, the following matrices such that $\pi_{i j}(h) \in\left[\pi_{i j, 1}, \pi_{i j, 2}\right]$ are established from Remark 3:

$$
\begin{aligned}
& {\left[\pi_{i j, 1}\right]_{i, j \in \mathbb{N}_{3}^{+}}=\left[\begin{array}{ccc}
-8.6880 & 0.0384 & 0.0896 \\
0.0192 & -4.3420 & 0.0448 \\
0.0128 & 0.0299 & -2.8951
\end{array}\right],} \\
& {\left[\pi_{i j, 2}\right]_{i, j \in \mathbb{N}_{3}^{+}}=\left[\begin{array}{ccc}
-0.1280 & 2.6064 & 6.0816 \\
1.3026 & -0.0640 & 3.0394 \\
0.8685 & 2.0266 & -0.0427
\end{array}\right] .}
\end{aligned}
$$

Figure 2(a) shows the mode evolution generated from the stochastic setting. Besides, from Theorem 6, the following control gains are obtained:

$$
\begin{aligned}
& F_{1}=\left[\begin{array}{ll}
0.2654 & -0.0063
\end{array}\right], \\
& F_{2}=\left[\begin{array}{ll}
-0.7178 & -0.1854
\end{array}\right], \\
& F_{3}=\left[\begin{array}{ll}
-1.9513 & 0.6293
\end{array}\right] .
\end{aligned}
$$

Figure 2(b) shows the behavior of the state response coupled with the mode transition depicted in Figures 2(a) and 2(c) which presents the Monte Carlo simulation result of the settling time for 5000 different mode transition configurations, where its mean value and standard deviation are 12.2979 and 3.126, respectively. From Figure 2(b), it can be seen that the states converge from the initial condition $(3.0,-5.0)$ to the equilibrium point as time increases. Consequently, Theorem 6 provides an applicable method for deriving a relaxed stabilization condition for S-MJSs with uncertain probability intensities.

Example 2. Consider the following system representing an inverted pendulum: for $x_{0}=(1,-1,0.5)$ and $\zeta_{0}=3$ :

$$
\begin{aligned}
\dot{x}_{1}(t) & =x_{2}(t), \\
\dot{x}_{2}(t) & =\frac{g}{l} \sin x_{1}(t)+\frac{N k_{m}}{m l^{2}} x_{3}(t), \\
L_{a} \dot{x}_{3}(t) & =k_{b} N x_{2}(t)-R(\zeta(t)) x_{3}(t)+\mathbf{q}(u(t)),
\end{aligned}
$$

where $x_{1}(t)$ is the angle of the inverted pendulum, $x_{2}(t)$ is the angular velocity, $x_{3}(t)$ is the input current, $u(t)$ is the control input voltage, $g$ is the acceleration due to gravity, $m$ and $l$ are the mass and length, respectively, $k_{b}$ and $k_{m}$ are the back emf constant and motor torque constant, respectively, $L_{a}$ and $R(\zeta(t))$ are the inductance and resistance of the DC motor, respectively, and $N$ is the gear ratio. Here, we set $L_{a}=1$, $g=9.8, l=1, m=1, N=10, K_{m}=K_{b}=0.1$, and

$$
R_{i}=R(\zeta(t)=i)= \begin{cases}2, & \text { for } \zeta(t)=1 \\ 1, & \text { for } \zeta(t)=2 \\ 0.5, & \text { for } \zeta(t)=3\end{cases}
$$

Then, the linearized model of (67) is given by

$$
\begin{aligned}
& A_{i}=A(\zeta(t)=i)\left[\begin{array}{ccc}
0 & 1 & 0 \\
9.8 & 0 & 1 \\
0 & 1 & -R_{i}
\end{array}\right], \\
& B_{1}=B_{2}=B_{3}=\left[\begin{array}{l}
0 \\
0 \\
1
\end{array}\right],
\end{aligned}
$$




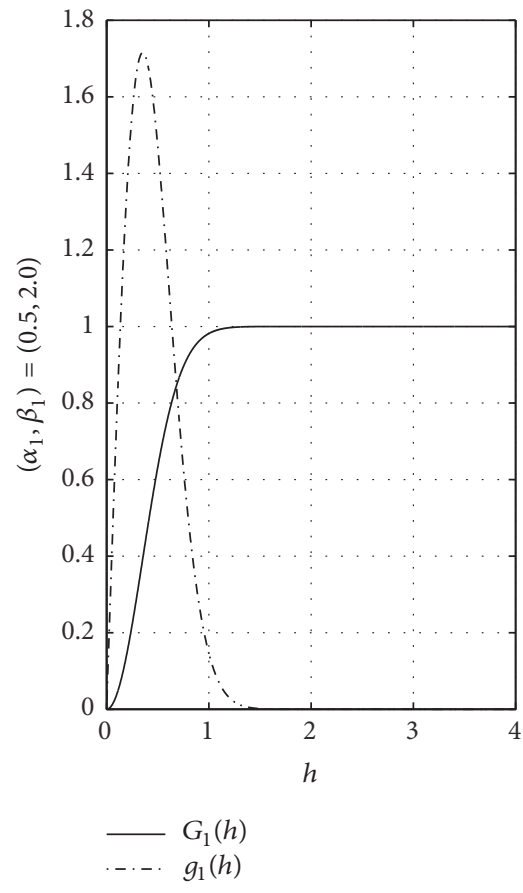

(a)

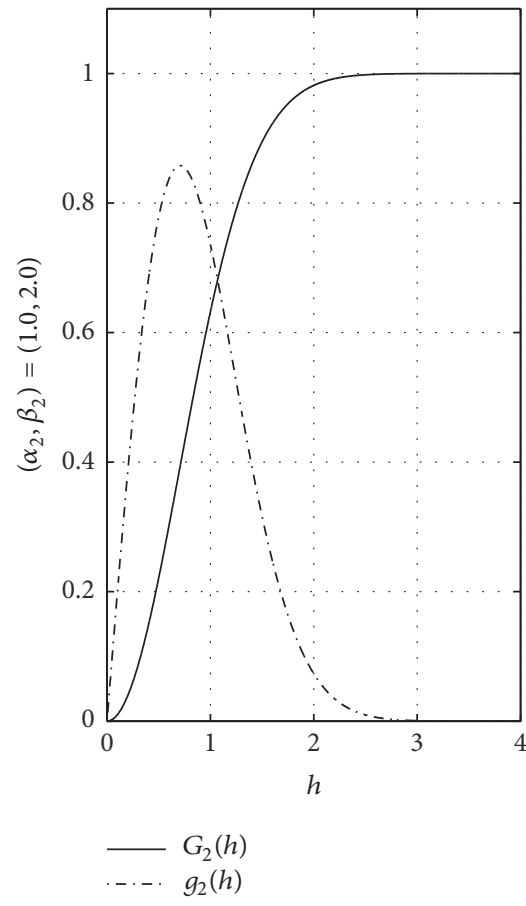

(b)

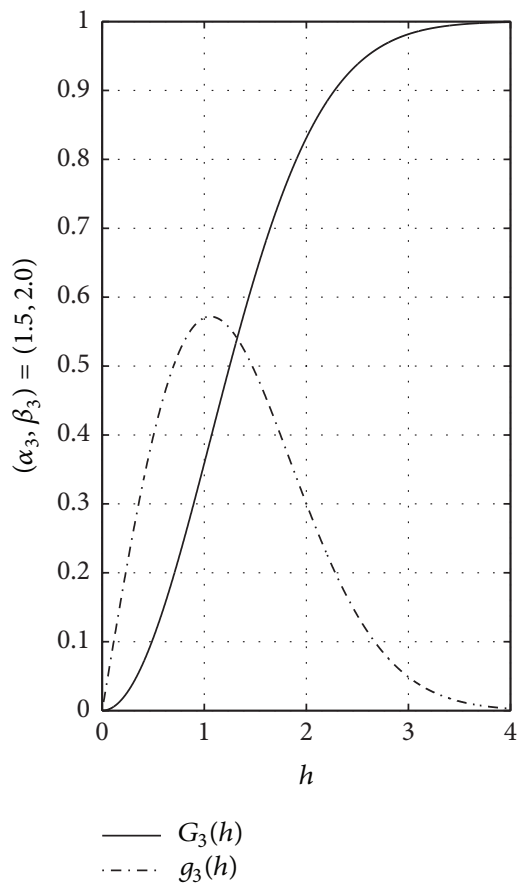

(c)

FIGURE 1: Cumulative distribution functions $G_{i}(h)$ (solid line) and probability distribution functions $g_{i}(h)$ (dash-dotted line). 


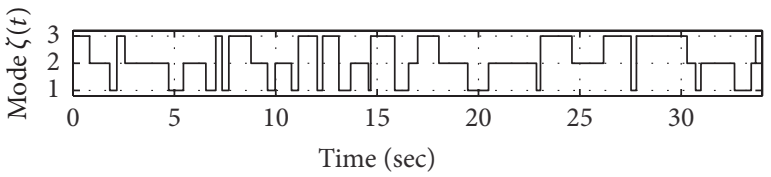

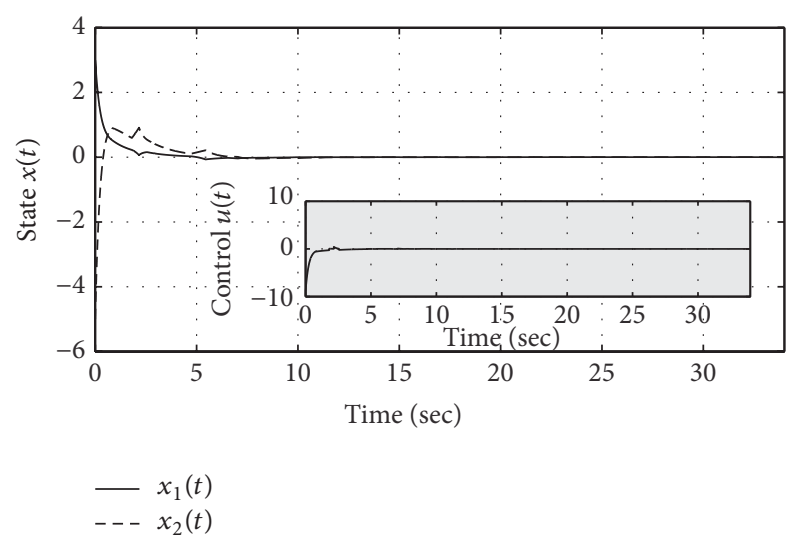

(b)

(a)

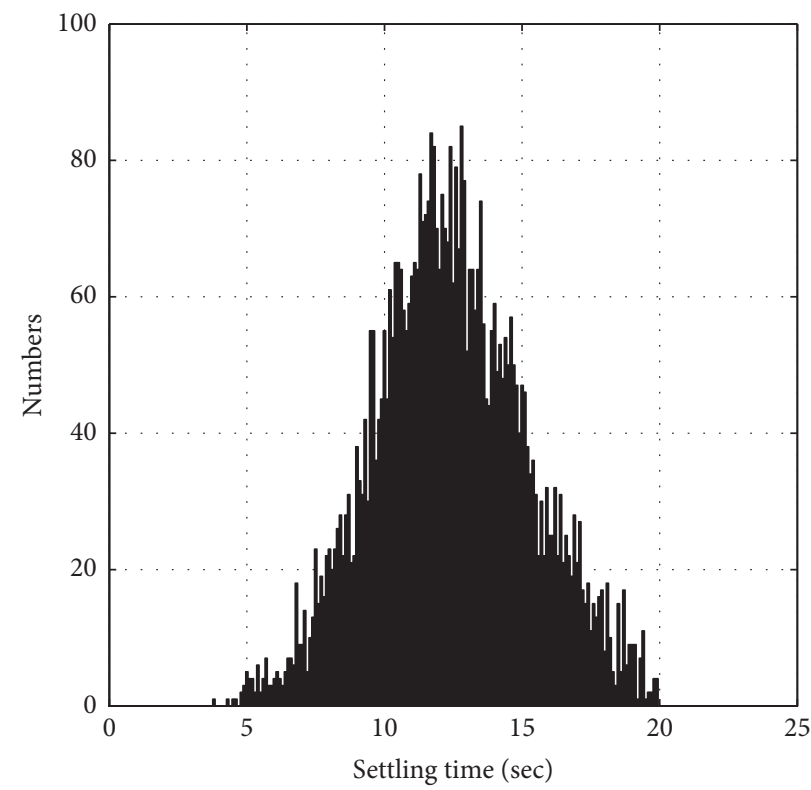

(c)

FiguRE 2: (a) Mode evolution, (b) state response and control input, and (c) Monte Carlo simulation results.

Besides, the matrices $\left[\pi_{i j, 1}\right]_{i j \in \mathbb{N}_{3}^{+}}$and $\left[\pi_{i j, 2}\right]_{i j \in \mathbb{N}_{3}^{+}}$such that $\pi_{i j}(h) \in\left[\pi_{i j, 1}, \pi_{i j, 2}\right]$ are taken to be the same as in Example 1. Thereupon, Theorem 7 provides the following control gains:

$$
\begin{aligned}
& F_{1}=\left[\begin{array}{lll}
-2.7348 & -3.1067 & 2.1744
\end{array}\right], \\
& F_{2}=\left[\begin{array}{lll}
-4.7643 & -3.6490 & 0.5326
\end{array}\right], \\
& F_{3}=\left[\begin{array}{lll}
-243.8434 & -79.7086 & -12.9784
\end{array}\right] .
\end{aligned}
$$

Figure 3 shows the behavior of the state response for the mode transition generated according to $\left(\alpha_{1}, \beta_{1}\right)=(0.5,2.0)$, $\left(\alpha_{2}, \beta_{2}\right)=(1.0,2.0)$, and $\left(\alpha_{3}, \beta_{3}\right)=(1.5,2.0)$. Here, the control input $v_{i}(t)$ is designed in accordance with (56), and the quantization level is assumed to be $\delta=0.1$. From Figure 3 , it can be seen that the states converge from the initial-state condition $(1,-1,0.5)$ to the origin as time increases. Consequently, Theorem 7 provides a suitable mode-dependent control for the S-MJSs with input quantization errors as well as uncertain probability intensities.

\section{Concluding Remarks}

The issue of stability analysis and control synthesis for S-MJSs with uncertain probability intensities has been addressed in this paper. Here, the boundary constraints of probability intensities have been totally reflected in the stabilization condition via a relaxation process established on the basis of time-varying transition rates. Furthermore, as an extension, the quantized control problem of S-MJSs has been addressed 


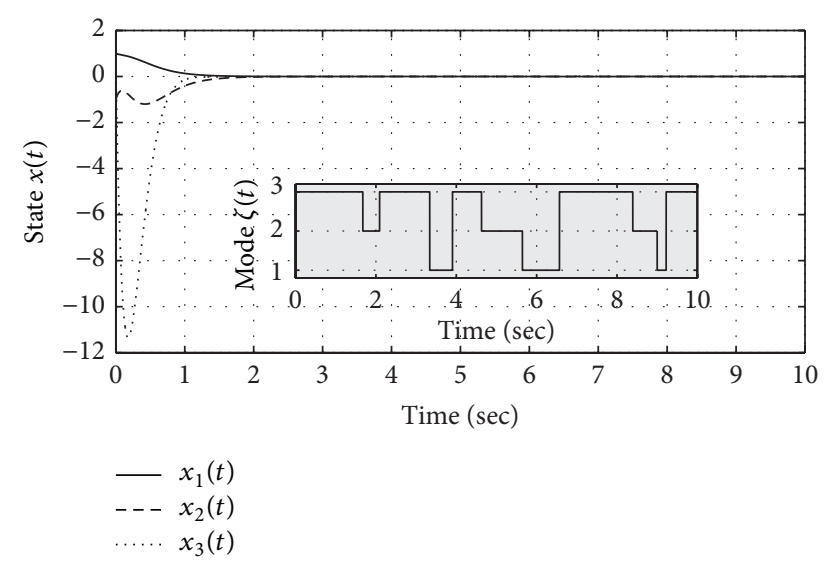

FIGURE 3: State response and mode evolution.

herein. Through simulation examples, the effectiveness of the proposed method has been shown.

\section{Competing Interests}

The authors declare that there is no conflict of interests regarding the publication of this paper.

\section{Acknowledgments}

This work was supported by the National Research Foundation of Korea Grant funded by the Korean Government (NRF-2015R1A1A1A05001131).

\section{References}

[1] E. K. Boukas, "Manufacturing systems: LMI approach," IEEE Transactions on Automatic Control, vol. 51, no. 6, pp. 1014-1018, 2006.

[2] F. Martinelli, "Optimality of a two-threshold feedback control for a manufacturing system with a production dependent failure rate," IEEE Transactions on Automatic Control, vol. 52, no. 10, pp. 1937-1942, 2007.

[3] H. Joo and S. H. Kim, " $\mathscr{H}_{\infty}$ LPV control with pole placement constraints for synchronous buck converters with piecewiseconstant loads," Mathematical Problems in Engineering, vol. 2015, Article ID 686857, 8 pages, 2015.

[4] P. Seiler and R. Sengupta, "An $H_{\infty}$ approach to networked control," IEEE Transactions on Automatic Control, vol. 50, no. 3, pp. 356-364, 2005.

[5] Y. Shi and B. Yu, "Output feedback stabilization of networked control systems with random delays modeled by Markov chains," IEEE Transactions on Automatic Control, vol. 54, no. 7, pp. 1668-1674, 2009.

[6] J. Wu and T. Chen, "Design of networked control systems with packet dropouts," IEEE Transactions on Automatic Control, vol. 52, no. 7, pp. 1314-1319, 2007.

[7] L. Zhang, Y. Shi, T. Chen, and B. Huang, "A new method for stabilization of networked control systems with random delays," IEEE Transactions on Automatic Control, vol. 50, no. 8, pp. 11771181, 2005.
[8] S. W. Yun and S. H. Kim, "Efficient approach to stability analysis of discrete-time systems with time-varying delay," Mathematical Problems in Engineering, vol. 2015, Article ID 813123, 9 pages, 2015.

[9] L. Li, V. A. Ugrinovskii, and R. Orsi, "Decentralized robust control of uncertain Markov jump parameter systems via output feedback," Automatica, vol. 43, no. 11, pp. 1932-1944, 2007.

[10] I. Matei and J. S. Baras, "A linear distributed filter inspired by the Markovian jump linear system filtering problem," Automatica, vol. 48, no. 8, pp. 1924-1928, 2012.

[11] J. Dong and G.-H. Yang, "Robust $H_{2}$ control of continuous-time Markov jump linear systems," Automatica, vol. 44, no. 5, pp. 1431-1436, 2008.

[12] H. Zhao, Q. Chen, and S. Xu, " $H_{\infty}$ guaranteed cost control for uncertain Markovian jump systems with mode-dependent distributed delays and input delays," Journal of the Franklin Institute-Engineering and Applied Mathematics, vol. 346, pp. 945-957, 2009.

[13] H. Li, Q. Zhou, B. Chen, and H. Liu, "Parameter-dependent robust stability for uncertain Markovian jump systems with time delay," Journal of the Franklin Institute-Engineering and Applied Mathematics, vol. 348, no. 4, pp. 738-748, 2011.

[14] E. K. Boukas, " $H_{\infty}$ control of discrete-time Markov jump systems with bounded transition probabilities," Optimal Control Applications \& Methods, vol. 30, no. 5, pp. 477-494, 2009.

[15] M. Shen and G.-H. Yang, " $H_{2}$ state feedback controller design for continuous Markov jump linear systems with partly known information," International Journal of Systems Science, vol. 43, no. 4, pp. 786-796, 2012.

[16] J. Xiong and J. Lam, "Robust $H_{2}$ control of Markovian jump systems with uncertain switching probabilities," International Journal of Systems Science, vol. 40, no. 3, pp. 255-265, 2009.

[17] L. Zhang and E.-K. Boukas, "Stability and stabilization of Markovian jump linear systems with partly unknown transition probabilities," Automatica, vol. 45, no. 2, pp. 463-468, 2009.

[18] S. H. Kim, "Less conservative stabilization conditions for Markovian jump systems with partly unknown transition probabilities," Journal of the Franklin Institute. Engineering and Applied Mathematics, vol. 351, no. 5, pp. 3042-3052, 2014.

[19] S. H. Kim, "Control synthesis of Markovian jump fuzzy systems based on a relaxation scheme for incomplete transition probability descriptions," Nonlinear Dynamics, vol. 78, no. 1, pp. 691701, 2014.

[20] S. H. kim, "Delay-dependent stability analysis for singular Markovian jump systems with incomplete transition probabilities," Journal of the Franklin Institute-Engineering and Applied Mathematics, vol. 352, no. 1, pp. 236-247, 2015.

[21] Z. Hou, J. Luo, and P. Shi, "Stochastic stability of linear systems with semi-Markovian jump parameters," The ANZIAM Journal, vol. 46, no. 3, pp. 331-340, 2005.

[22] Z. Hou, J. Luo, P. Shi, and S. K. Nguang, "Stochastic stability of Ito differential equations with semi-Markovian jump parameters," IEEE Transactions on Automatic Control, vol. 51, no. 8, pp. 1383-1387, 2006.

[23] L. Zhang, " $H_{\infty}$ control of a class of piecewise homogeneous Markov jump linear systems," in Proceedings of the 7th Asian Control Conference (ASCC '09), pp. 197-202, Hong Kong, August 2009.

[24] C. Schwartz, Control of semi-Markov jump linear systems with application to the bunch-train cavity interaction [Ph.D. thesis], Northwestern University, 2003. 
[25] J. Huang and Y. Shi, "Stochastic stability of semi-Markov jump linear systems: an LMI approach," in Proceedings of the 50th IEEE Conference on Decision and Control and European Control Conference (CDC-ECC '11), pp. 4668-4673, Orlando, Fla, USA, December 2011.

[26] X. Liu, X. Yu, G. Ma, and H. Xi, "On sliding mode control for networked control systems with semi-Markovian switching and random sensor delays," Information Sciences, vol. 337-338, pp. 44-58, 2016.

[27] J. Huang and Y. Shi, "Stochastic stability and robust stabilization of semi-Markov jump linear systems," International Journal of Robust and Nonlinear Control, vol. 23, no. 18, pp. 2028-2043, 2013.

[28] J. Huang and Y. Shi, " $H_{\infty}$ stability-feedback control for semiMarkovian jump linear systems with time-varying delays," Journal of Dynamic Systems, Measurement, and Control, vol. 135, no. 4, Article ID 041012, 2013.

[29] F. Li, L. Wu, P. Shi, and C.-C. Lim, "State estimation and sliding mode control for semi-Markovian jump systems with mismatched uncertainties," Automatica, vol. 51, pp. 385-393, 2015.

[30] H. Shen, Z.-G. Wu, and J. H. Park, "Reliable mixed passive and $H_{\infty}$ filtering for semi-Markov jump systems with randomly occurring uncertainties and sensor failures," International Journal of Robust and Nonlinear Control, vol. 25, no. 17, pp. 32313251, 2015.

[31] S. H. Kim, "Multi-stage control strategy for quantised feedback control systems," IET Control Theory \& Applications, vol. 7, no. 4, pp. 584-593, 2013.

[32] S. W. Yun, S. H. Kim, and J. Y. Park, "Asymptotic stabilization of continuous-time linear systems with input and state quantizations," Mathematical Problems in Engineering, vol. 2014, Article ID 947164, 6 pages, 2014.

[33] A. Svishchuk, Random Evolutions and Their Applications: New Trends, Kluwer Academic, Norwell, Mass, USA, 2000.

[34] S. Boyd, L. E. Chauoi, E. Feron, and V. Balakrishan, Linear Matrix Inequalities in Systems and Control Theory, SIAM, Philadelphia, Pa, USA, 1994. 


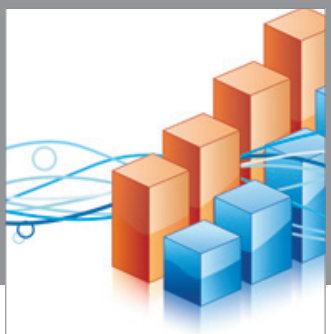

Advances in

Operations Research

vatem alat4

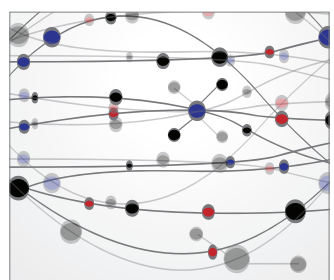

\section{The Scientific} World Journal
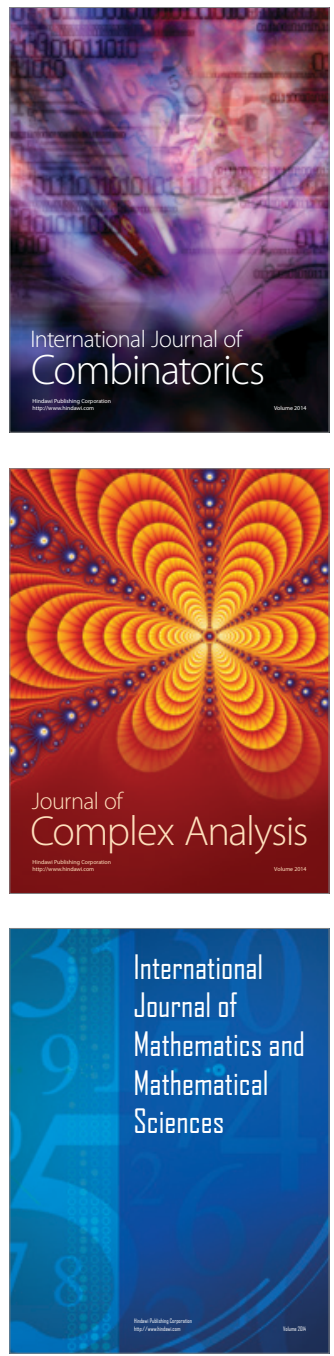
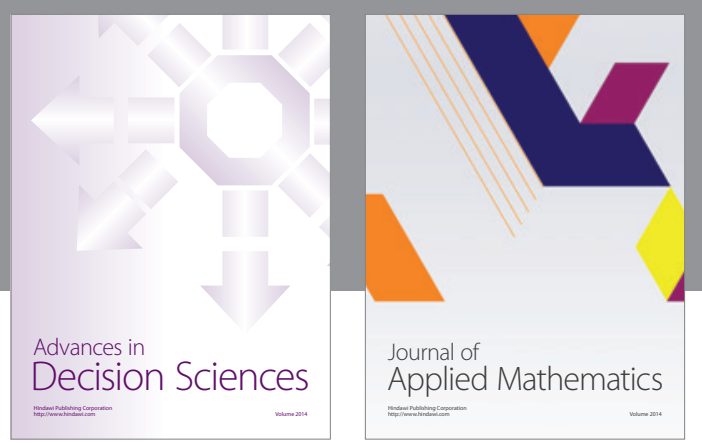

Algebra

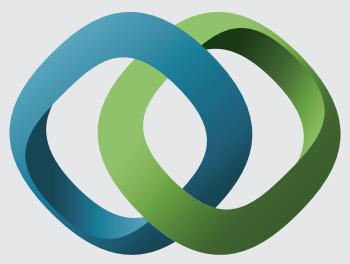

\section{Hindawi}

Submit your manuscripts at

http://www.hindawi.com
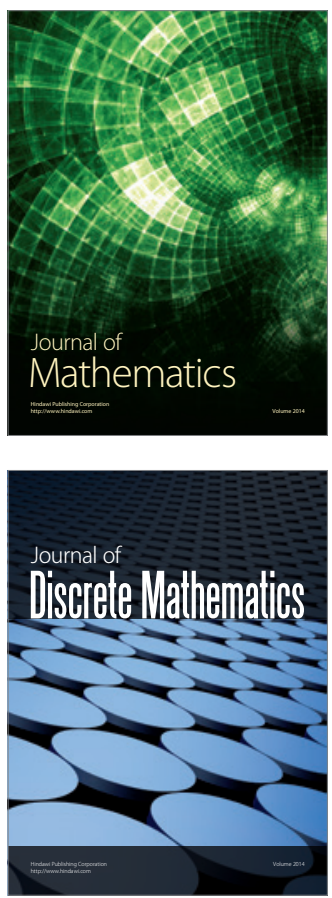

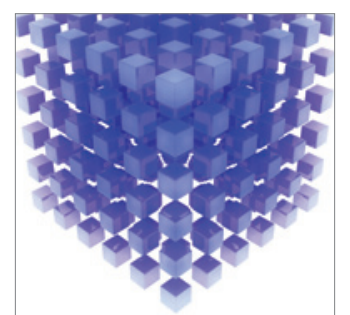

Mathematical Problems in Engineering
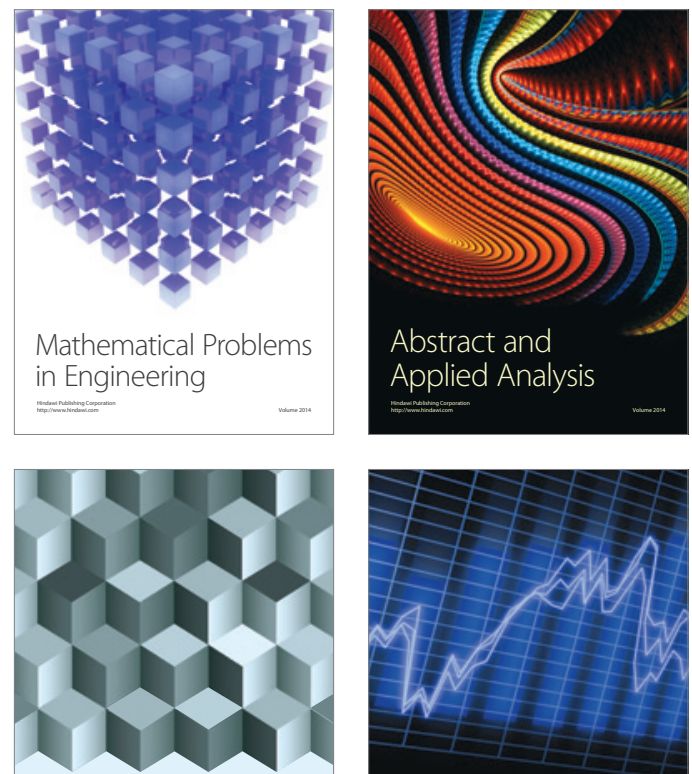

Journal of

Function Spaces

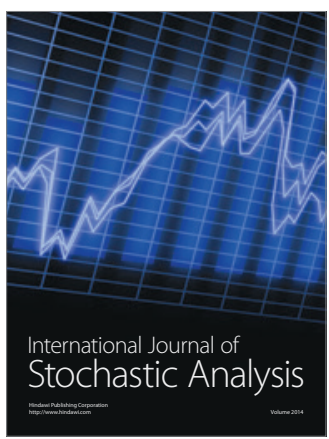

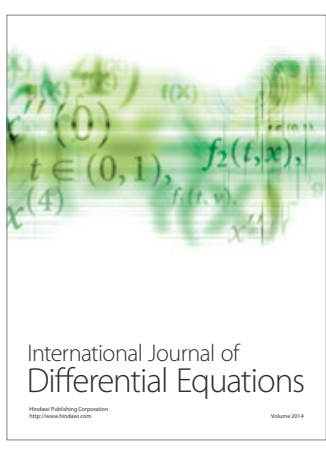
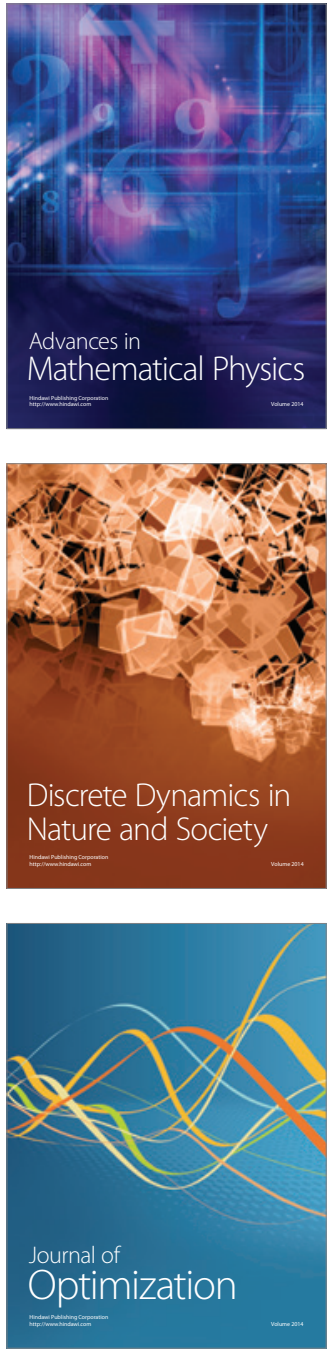